\title{
Uptake and Intracellular Fate of Peptide Surface- Functionalized Silica Hybrid Magnetic Nanoparticles In Vitro
}

\author{
Reinaldo G. Digigow, Dimitri Vanhecke, Barbara Rothen-Rutishauser, Martin J.D. Clift,* \\ and Alke Petri-Fink**
}

\begin{abstract}
Recently, the use of nanomaterials as intracellular targeting tools for theranostics has gained heightened interest. Despite the clear advantages posed by surface-functionalized nanoparticles (NPs) in this regard, limited understanding currently exists due to difficulties in reliably synthesizing NPs with surface functionalizations adequate for use in such applications, as well as the manner of analytics used to assess the cellular uptake and intracellular localization of these NPs. In the present study, two key surface functionalities (a nuclear localization sequence (NLS) and integrin-ligand (CRGD)) are attached to the surface of multifunctional, silica hybrid magnetic nanoparticles (SHMNPs) containing a polyethylene glycol (PEG) polymer coating using a well-described, reliable, and reproducible microreactor set-up. Subsequent analytical interpretation, via laser scanning confocal, transmission electron and dark-field microscopy, as well as flow cytometry, of the interaction of SHMNPs-PEG-cRGD-NLS with macrophage (J774A.1) and epithelial (HeLa) cells shows internalization of the SHMNPs-PEG-cRGD-NLS in both cell types up to $24 \mathrm{~h}$ after $20 \mu \mathrm{g} \mathrm{mL}^{-1}$ exposure, as well as increasing aggregation inside of vesicles over this time period. The findings of this study show that by incorporating a variety of state-of-the-art analytical and imaging approaches, it is possible to determine the specific effectiveness of surface peptide and ligand sequences upon multifunctional SHMNPs.
\end{abstract}

\section{Introduction}

The development and use of nanomaterials as advantageous medical-based applications have shown increased potential in the last decade, particularly in the field of diagnostics and therapeutics. $^{[1]}$ For many biomedical applications, the ability

Dr. R. G. Digigow, Dr. D. Vanhecke,

Prof. B. Rothen-Rutishauser, Dr. M. J. D. Clift,

Prof. A. Petri-Fink

Adolphe Merkle Institute

University of Fribourg

Route de l'Ancienne Papeterie

P.O. Box 209, 1723, Marly 1, Switzerland

E-mail: martin.clift@unifr.ch; alke.fink@unifr.ch

Dr. R. G. Digigow, Prof. A. Petri-Fink

Department of Chemistry

University of Fribourg

Chemin du Musée 9, 1700 Fribourg, Switzerland to deliver precise payloads to relevant cell types, as well as specific intracellular organelles of interest, is essential. ${ }^{[2]}$ To achieve this, selected nanomaterials, such as quantum dots, ${ }^{[3]}$ gold $^{[4]}$ or polystyrene $e^{[5]}$ nanoparticles (NPs) have previously been studied. However, biocompatible ${ }^{[6]}$ superparamagnetic iron oxide nanoparticles (SPIONs) are conceived as beneficial, ${ }^{[7-10]}$ alternative targeting tools compared to other nanomaterials ${ }^{[11]}$ as they are easily synthesized with a high reproducibly. ${ }^{[12]}$ In order to allow for efficient cellular internalization and targeting of specific intracellular organelles while ensuring colloidal stability, the SPIONs' surface has to be functionalized with both stabilizers (e.g., synthetic polymers) and targeting moieties (e.g., surface peptides and ligands). ${ }^{[13]}$ To achieve this, multi-step liquid-phase NP surface derivatization is required. Yet, this is highly challenging, since each coupling reaction is typically carried out in a specific buffer, followed by a purification step such as magnetic sedimentation, size exclusion chromatography, or dialysis. ${ }^{[14,15]}$ Repeated magnetic sedimentation may cause irreversible agglomeration of the NPs, ${ }^{[16]}$ while size exclusion chromatography can result in unwanted dilution of the suspension and loss of the material on the column after each purification step.

Thus, in the past decade, in order to try and overcome these issues, the basis of numerous studies has been to determine the most efficient manner to synthesize multifunctional NPs with a specific bio-affinity towards intracellular organelles, specifically the cell nucleus (e.g., for cancer therapy). ${ }^{[17]}$ In a notable study, Tkachenko and co-workers ${ }^{[18]}$ reported that the nuclear compartment of both epithelial and hepatocyte cells could be actively targeted by gold ( $\mathrm{Au}$ ) NPs, although only after surface assembly with a nuclear localization signal (NLS) peptide conjugated to bovine serum albumin. It was further shown that access of the Au NPs into the nucleus was both time $(\leq 3 \mathrm{~h})$ and cell-type dependent (i.e., fibroblasts, epithelial, and hepatocyte cells). ${ }^{[18]}$ In another study, Chen et al. ${ }^{[19]}$ recently demonstrated the enhancement of the cellular uptake of liposomes doped with doxorubicin both in vivo (Sprague-Dawley rats) 
and in vitro (glicoma cells) when the liposomes also exhibited the integrin-ligand Arg-Gly-Asp-D-Phe-Cys (RGD) in its cyclic form (cRGD) on their surface; cRGD is well known to enhance receptor-specific uptake by, and targeted gene delivery to tumor cells due to their overexpression of surface integrins. ${ }^{[20]}$ The presence of liposomes within human glioma U87MG cells was confirmed only by flow cytometry. Chen et al. ${ }^{[19]}$ reported that the surface chemistry used enabled an integrin-specific $(\alpha v \beta 3$ and $\alpha v \beta 5$ ) endocytosis and therefore, a subsequent efficient drug release.

Despite these reported findings, it is difficult to comprehensively determine the precise effectiveness of the different surface peptide and ligand complexes' ability to target intracellular organelles due to the NPs themselves, as well as the limited, complimentary analytical techniques employed. Therefore, the aim of the current study was to i) utilize a previously developed magnetic microreactor to reproducibly synthesize highly complex magnetic NPs carrying two peptides (NLS and cRGD) and ii) determine, unambiguously, the uptake and intracellular localization of these NPs in two different cell types by using a combination of complementary analytical and imaging techniques.

\section{Results and Discussion}

\subsection{Particle Synthesis and Characterization}

To ensure a fast and efficient response to the external magnetic field, ${ }^{[11]}$ a large number of presynthesized individual SPIONs were incorporated in a silica matrix, as previously reported. ${ }^{[21]}$ Briefly, co-condensation of 3-aminopropyltriethoxysilane (APTES) and tetraethyl orthosilicate (TEOS) in the presence of SPIONs was used to synthesize amine-functionalized silica coated SPIONs (SHMNPs) using methods as described before. ${ }^{[21]}$

Coupling of the peptides on the magnetic NPs was accomplished using heterobifunctional polyethylene glycol (PEG) as a cross-linker. PEGylation was performed to enhance NP colloidal stability, in particular in serum supplemented cell culture medium. ${ }^{[22]}$ In order to assure a continuous and highly controlled functionalization process, SHMNPs were immobilized as a stationary solid phase in a column using a high-gradient permanent magnetic field. This reactor has previously been developed for research laboratories working with magnetic NPs for easy, efficient, and reproducible magnetic NP surface functionalization. ${ }^{[11]}$ All SPION surface derivations and purification steps were carried out using this magnetic reactor setup. Four different NPs with increasing surface complexity were manufactured and characterized; bare SPIONs, ${ }^{[23]}$ SHMNPs, PEGylated silica beads with embedded SPIONs (SHMNPsPEG), and cRGD in parallel with the fluorescently labeled (i.e., AlexaFluor488) nuclear targeting peptide-derivatized SPIONs (SHMNPs-PEG-cRGD-NLS). In several studies, it was demonstrated that such multifunctional magnetic NPs could only be reproducibly synthesized in such magnetic reactors; standard liquid-phase syntheses led to misrepresentative outcomes and often resulted in aggregation. ${ }^{[12,24]}$ Their hydrodynamic sizes and zeta potentials, obtained by dynamic light scattering (DLS), are summarized in the table in Figure 1B.

A representative transmission electron microscopy (TEM) image of the SHMNPs is shown in Figure 1C. The spherical, contrast-rich single SPIONs (as denoted by the arrow in Figure 1C) are surrounded by the weak electron scattering silica layer. Both the PEG polymer and the surface-attached peptides are unable to be resolved using TEM.

For PEGylation and peptide coupling, reaction conditions were optimized in terms of concentration, incubation time, and

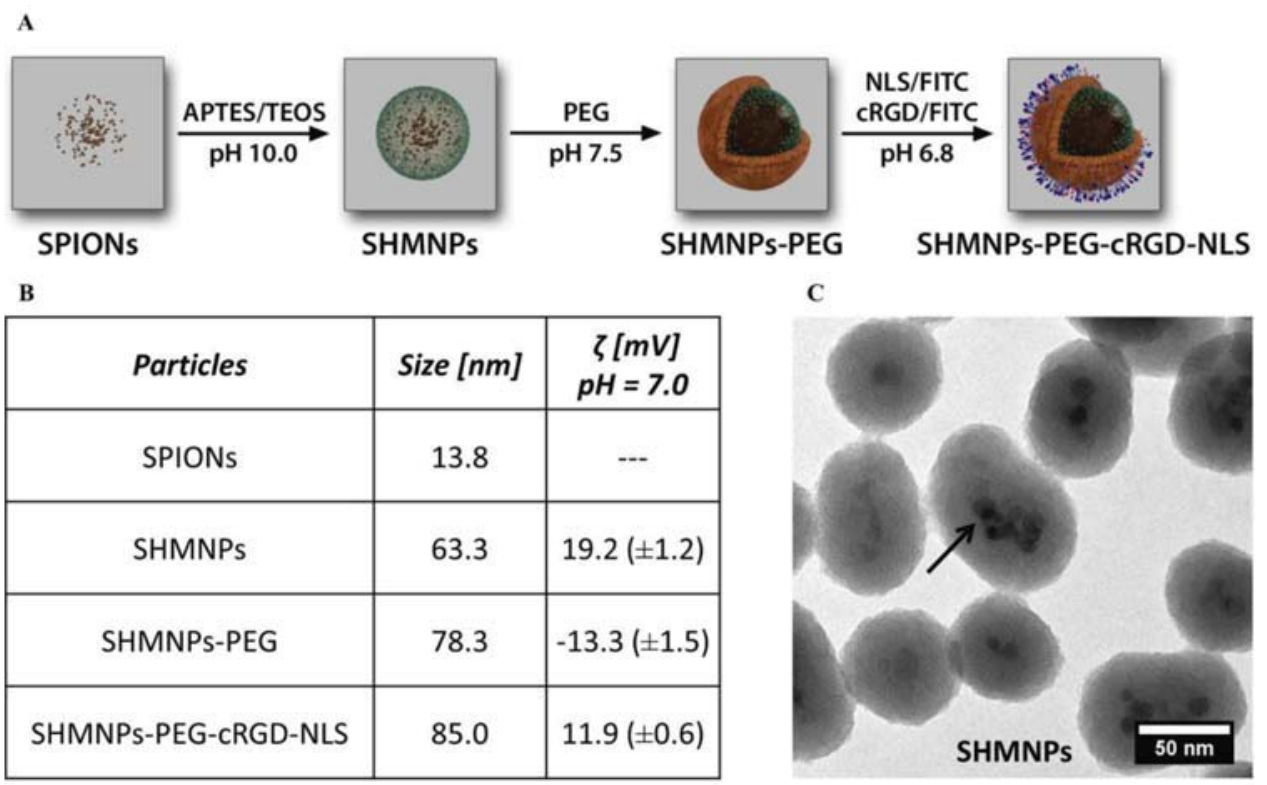

Figure 1. A) Scheme of the synthetic route of SHMNPs surface functionalization, B) Physicochemical characterization (mean diameter and zeta potential by dynamic light scattering), and C) representative transmission electron microscopy image showing SHMNPs (SPIONs in the center (black dots (indicated by the black arrow))) with the surrounding silica shell. 
re-circulation cycles to enhance colloidal stability. As expected, the initial zeta potential of $19.2 \pm 1.2 \mathrm{mV}$ for SHMNPs decreased significantly after PEGylation to $-13.3 \pm 1.5 \mathrm{mV}$ (Figure 1B), which is in agreement with previous studies. ${ }^{[11]}$ Nonetheless, subsequent peptide coupling increased the zeta potential to $11.9 \pm 0.6 \mathrm{mV}$. The nuclear targeting peptide employed contains the heptamer functional site PKKKRKVGC ${ }^{[25]}$ derived from the SV40 large T-antigen NLS that is responsible for nuclear targeting, where the $\mathrm{GC}$ residues were engineered into the peptide to allow a spacer (glycine) and a C-terminal cysteine for facile conjugation to the functionalized SPIONs. The ligand Arg-GlyAsp-D-Phe-Cys (RGD) in its cyclic form (cRGD) is well known to enhance receptor-specific uptake and both used cell types are known to express the specific integrins for CRGD. [26-29]

In parallel, the mean NP hydrodynamic size increased from 63.3 (SHMNPs) to $78.3 \mathrm{~nm}$ upon PEGylation, which can be explained by the presence of the PEG layer (MW of ca. $5000 \mathrm{Da}$ ), as previously reported. ${ }^{[30]}$ This diameter slightly increased upon peptide coupling and a mean diameter of $85 \mathrm{~nm}$ (Figure 1B) was obtained for the fully functionalized NPs (i.e., SHMNPs-PEG-cRGD-NLS).

As a control, dye-labeled analogs using AlexaFluor488-peptide or SAMSA-fluorescein-PEG have been synthesized to further demonstrate the presence of the polymer and peptides, respectively, and to obtain a rough estimate about their quantity. The fluorescence spectra show an emission at $515 \mathrm{~nm}$, which is characteristic for the SAMSA-fluorescein dye. ${ }^{[31]}$ A PEG grafting density of approximately 200 PEG molecules per NP can be estimated, corresponding to a coverage density of 0.01 PEG chains per $\mathrm{nm}^{2}$, which is consistent with previous studies. ${ }^{[32]}$ The fluorescent tag of the peptide (AlexaFluor488) allowed for detection and estimation of cRGD coupling. On average, ca. 20 cRGD molecules were coupled per SHMNP, as determined by fluorescent spectroscopy.

\subsection{Cellular Interactions of SHMNP-PEG-cRGD-NLS}

It has previously been reported that the cellular uptake of NPs is strongly dependent upon the cell type used. ${ }^{[33]}$ Since current literature intensely focuses upon epithelial cell types, ${ }^{[18]}$ the cervical carcinoma epithelial cell-line (HeLa) was used as a model. In addition, the understanding of how functionalized SPIONS interact with immune cells remains limited. Therefore, the J774A.1 "macrophage-like" cell-line was also used in the present study. From literature, it is well known that both cell types express the specific integrins for cRGD. ${ }^{[26-29]}$

\subsubsection{Uptake and Cytotoxicity of SHMNPs-PEG-cRGD-NLS}

The cellular interaction of the SHMNPs-PEG-cRGD-NLS was studied by laser scanning microscopy (LSM) (Figure 2). Both macrophage and epithelial cells showed internalization of the SHMNPs-PEG-cRGD-NLS after $2 \mathrm{~h}$ exposure (Figure 2a). The J774A.1 macrophage-like cells further displayed a continuous and increased level of internalization of the NPs up to $24 \mathrm{~h}$ at a concentration of $20 \mu \mathrm{g} \mathrm{mL}^{-1}$ (Figure 2a; Figure 1, Supporting Information). Examination of the interaction of
SHMNPs-PEG-cRGD-NLS with the HeLa cells showed less uptake compared to the J774A.1 cells of the SHMNPs-PEGcRGD-NLS over the $24 \mathrm{~h}$ period at the same concentration (Figure 2a; Figure 1, Supporting Information).

Investigation of the cellular uptake of SHMNPs-PEG-cRGDNLS by flow cytometry supported the findings of the LSM analysis, with an increase in J774A.1 cellular fluorescence seen at both 6 and $24 \mathrm{~h}$ compared to that observed after $2 \mathrm{~h}$ after $20 \mu \mathrm{g} \mathrm{mL}$ exposure (Figure 2b). Similarly, the intracellular fluorescence was found to be constant in the epithelial HeLa cells over the $24 \mathrm{~h}$ period (Figure $2 \mathrm{~b}$ ), as shown with LSM (Figure 2a).

The differences observed can be attributed to the fact that epithelial cells do not readily internalize foreign objects, while macrophage cells are considered "professional phagocytes" and primarily engulf any extraneous material. ${ }^{[34]}$ Furthermore, the observation that the SHMNPs-PEG-cRGD-NLS actively interacted with the macrophage cells is in congruence with previous literature that has reported the continuous uptake of NPs such as semiconductor quantum dots or polystyrene NPs with J774A.1 macarophage cells over time, ${ }^{[35]}$ thus highlighting the dynamic interaction between NPs and immune cells. Other studies have also shown that NPs of various shapes, materials, or surfaces can interact with immune cells such as macrophages; ${ }^{[36-38]}$ however, since the cell types employed there are not the same as the ones used in the present study, a direct comparison between findings is difficult. It is also important to note, that the findings of the current study support those previously reported by Tkachenko and co-workers, ${ }^{[18]}$ who showed the efficacy of NP intracellular targeting to be highly dependent upon the cell type being used.

Due to the clear and efficient interaction of the SHMNPsPEG-cRGD-NLS with the J774A.1 macrophage cells, these cells were chosen for all subsequent experiments. To further define optimal experimental conditions, two exposure concentrations (20 and $100 \mu \mathrm{g} \mathrm{mL} \mathrm{m}^{-1}$ ) were tested. A clear concentrationdependent uptake of the SHMNPs-PEG-cRGD-NLS by the macrophage cells was observed over a $24 \mathrm{~h}$ period (Figure 2, Supporting Information). It was apparent, however, that following exposure to $100 \mu \mathrm{g} \mathrm{mL} \mathrm{m}^{-1}$ macrophage cells were severely overloaded (Figure 2, Supporting Information). These findings subsequently alluded to the use of the SHMNPs-PEG-cRGDNLS at the lower concentration of $20 \mu \mathrm{g} \mathrm{mL}^{-1}$ for all microscopic evaluation. It is also important to note that no cytotoxicity was observed in either cell type at both concentrations tested after $24 \mathrm{~h}$ suspension exposure (Figure 3, Supporting Information).

\subsubsection{Intracellular Localization of SHMNPs-PEG-CRGD-NLS}

Many studies in the past have focused on intracellular NP delivery. Typically, cellular localization of NP has been assessed by light microscopic methods using markers, ${ }^{[35]}$ such as fluorescent tags and, to a lesser extent, using the strong light scattering feature of noble metals or metal oxides in the dark-field mode. ${ }^{[39]}$ It is, however, difficult to make a clear assessment about the NPs intracellular localization, since the provided light microscopy often only displays 2D information. Due to the resolution limit of light microscopy, which is in the range of 200-500 nm, TEM, with a resolution range of Ångstrom to 

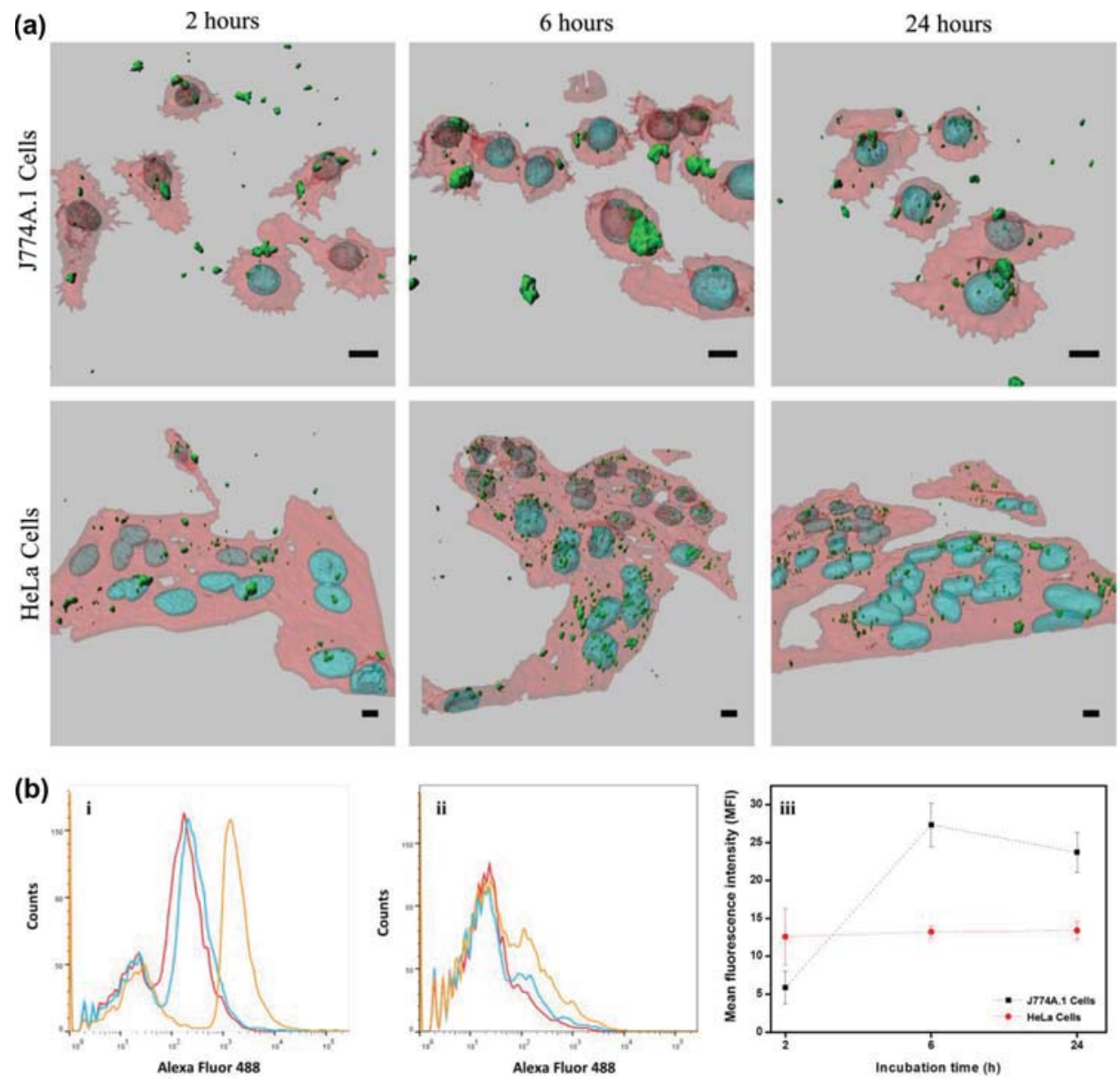

Figure 2. a) Laser scanning confocal microscopy (LSM) images showing the internalization of SHMNPs-PEG-cRGD-NLS in J774A.1 cells and HeLa cells after 2, 6, and $24 \mathrm{~h}$ exposure at $20 \mathrm{\mu g} \mathrm{mL} \mathrm{L}^{-1}$. Images show surface rendering of 3D stacks. Red color corresponds to F-actin. Cyan color indicates the nuclear region of cells (DAPI stain). Green fluorescence corresponds to the SHMNPs-PEG-cRGD-NLS. Scale bars represent $10 \mu \mathrm{m}$. b) Cellular association, as determined by flow cytometry, of SHMNPs-PEG-cRGD-NLS in i) J774A.1 cells and ii) HeLa cells after 2 (red), 6 (orange), and 24 (blue) $\mathrm{h}$ exposure at $20 \mu \mathrm{g} \mathrm{mL}^{-1}$. The negative control (media only) was set to the first logarithmic decade (data not shown). Image iii) shows the semiquantitation time-resolved cellular association of the SHMNPs-PEG-CRGD-NLS with each different cell type.

nanometer, is typically used for resolving single NPs. ${ }^{[40]}$ In the present manuscript, a combination of conventional TEM and dark-field microscopy was used to identify the intracellular localization of SHMNPS-PEG-cRGD-NLS and to validate the data obtained by LSM (Figure 2a). The visualization was complemented by hyperspectral mapping in dark-field microscopy as well as with elemental spectroscopy imaging (ESI) with TEM.

Hyperspectral imaging of a J774.1 macrophage cells exposed to SHMNPs-PEG-cRGD-NLS for $24 \mathrm{~h}$ is shown in Figure 3. The emitted fluorescence and scattering signals of the SHMNPsPEG-cRGD-NLS were well-defined and spots could be located i) near the cell membrane, ii) within the cell and iii) in the vicinity of the nucleus (two green fluorescent spots originating from SHMNP-PEG-cRGD-NLS, denoted by the arrowheads). Based on a cell-free SHMNPs sample (Figure 4, Supporting Information), a collection of typical spectral fingerprints of SHMNPs were collected and matched by spectral angle mapping with the recorded spectral data of the cell. The image and corresponding spectra of the detected SHMNPs-PEG-cRGD-NLS inside and attached to the macrophage cell (Figure 3D) show the pixels relevant to a representative SHMNPs spectral fingerprint. These spectral SHMNPs fingerprints overlap with the emitted fluorescence of the SHMNPs-PEG-cRGD-NLS (Figure 3A) and with the dark-field scattering signal of the SPIONs (Figure 3B). It is important to consider that since the signals recorded have a spatial extent of approximately hundreds of nanometers in the orthogonal plane, the precision in localization of the SHMNPSPEG-cRGD-NLS is limited. Nonetheless, the observed small red shift in the scattered wavelengths between clearly intracellular and extracellular NPs is noteworthy (Figure 3D), and can further be postulated as highlighting the aggregation of the NPs inside of the cells.

In the next step, TEM was applied to confirm the data obtained by LSM and dark-field microscopy. Figure 4 shows a TEM image of a representative J774A.1 macrophage cell after exposure to SHMNP-PEG-cRGD-NLS for $24 \mathrm{~h}$. Structural comparison of the round, dark objects inside a vesicle (Figure 4B) indicate that these are SHMNPs-PEG-cRGD-NLS: one, or a few electron dense central cores $\approx 6 \mathrm{~nm}$ in diameter surrounded by a weaker scattering structure with a diameter of $\approx 100 \mathrm{~nm}$. 

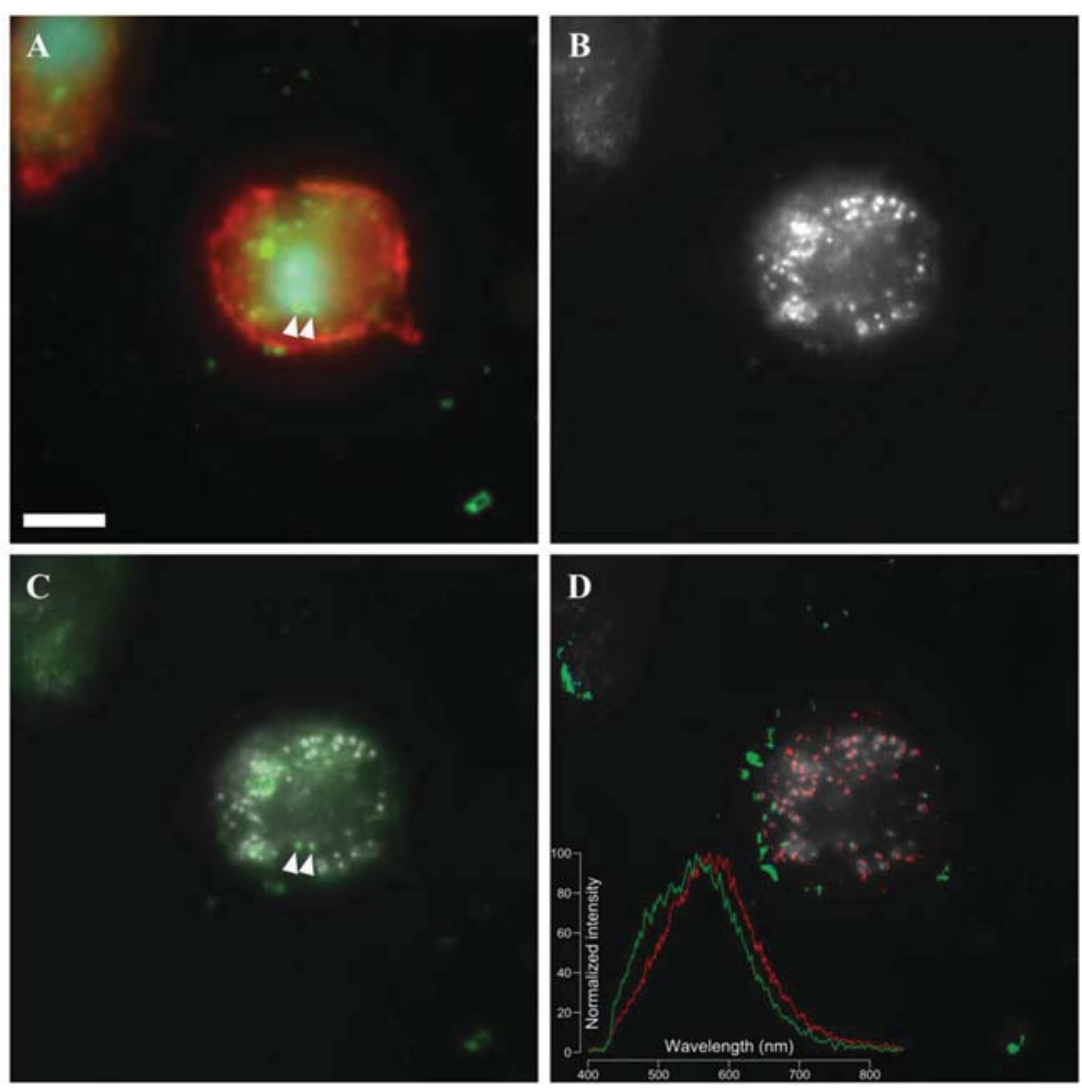

Figure 3. Fluorescence, dark-field, and hyperspectral imaging of J774A.1 cells after exposure to SHMNPs-PEG-cRGD-NLS for $24 \mathrm{~h}$ at $20 \mu \mathrm{g} \mathrm{mL} \mathrm{L}^{-1}$. A) Fluorescence signal showing F-actin (red), SHMNPS-PEG-cRGD-NLS (green), and nucleus (cyan). B) Dark-field image of constituting the same field of view as A. C) An overlay of the SHMNPs-PEG-CRGD-NLS fluorescence signal and the dark-field signal. D) Hyperspectral imaging of the same field of view and spectral angle mapping of the SHMNPs-PEG-CRGD-NLS. The spectra of the nanoparticles overlap with both the dark-field image and the SHMNPs-PEG-cRGD-NLS fluorescence. Scale bar represents $5 \mu \mathrm{m}$.

Silicon ( $\mathrm{Si}$ ) elemental mapping $\left(\mathrm{SiO}_{2}\right.$ is the major component of the SPION containing beads SHMNPs and otherwise absent in a cellular environment) and energy loss spectroscopy ( $\mathrm{Si}$ and Fe; Figure 5, Supporting Information) of the components within the cell proved these objects to be SHMNPs-PEG-cRGDNLS (Figure 3D).
This data set supports the notion that the SHMNPs-PEG-cRGD-NLS are aggregated inside a vesicular structure. Reasoning for this is, although the SHMNPs-PEG-cRGDNLS are normally well dispersed and stable, when present inside a cellular compartment

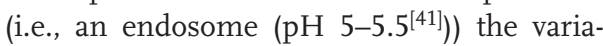
tion in $\mathrm{pH}$ can compromise their stability. This change in $\mathrm{pH}$, coupled with a large number of proteins and electrolytes, as compared with, for example, ultrapure water or phosphate buffered saline can result in aggregation of the NPs. ${ }^{[6]}$

\subsubsection{Impact of Surface Functionalization on Particle Uptake and Intracellular Fate}

The impact of different degrees of surface modifications of the NPs on their interaction with the J774A.1 cells was then investigated in order to validate the different functionalization steps performed with the microreactor set-up. Cells were either exposed to SHMNPs-PEG-NLS, SHMNPs-PEG-cRGD, or SHMNPs-PEG-cRGD-NLS for $24 \mathrm{~h}$ at $20 \mu \mathrm{g} \mathrm{mL} \mathrm{L}^{-1}$ (Figure 5).

A colocalization analysis of the NPs with the cell nuclei was subsequently performed with the "IMARISColoc" module in order to show any possible association with the nuclei. When the NLS peptide was present on the surface of the SHMNPs, NPs were taken up by the cells and a close association of the NPs with the nuclear region was observed (Figure 5A). It is well documented that the coupling of specific peptide sequences on NPs is advantageous in directing the system across the intracellular membranes. ${ }^{[42]}$ For example, Au NPs coupled with a number of different peptide sequences (e.g., NLS) were used before ${ }^{[18]}$ to target the nucleus, where it was shown that the NPs were located in different parts of the cell, including the nucleus, and their
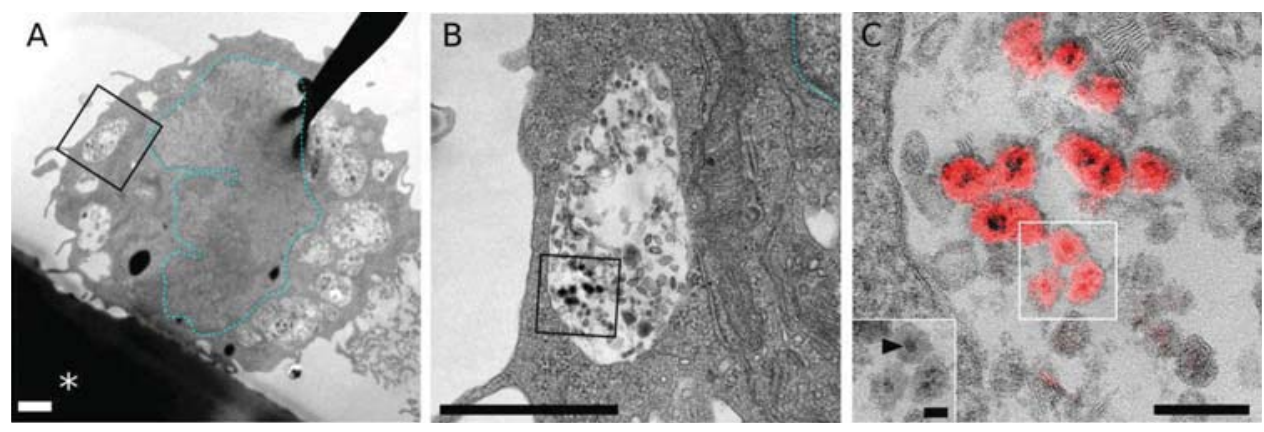

Figure 4. Transmission electron microscope (TEM) image of J774A.1 cells after exposure to SHMNP-PEG-cRGD-NLS for $24 \mathrm{~h}$ at $20 \mu \mathrm{gg} \mathrm{mL} \mathrm{L}^{-1}$. A) shows an overview of the J774A.1 cell, grown on an insert (*) and highlighting a membrane bound vesicle containing SHMNPs-PEG-CRGD-NLS in close association with the nucleus (cyan dotted line). B) is a higher magnification of the inset in (A), highlighting the morphology of the SHMNPs-PEG-cRGD-NLS. C) shows the silicon ( $\mathrm{Si}$ ) elemental map (red) of the inset in Figure 5B overlaid with the conventional TEM image. The inset shows three SHMNPs-PEGCRGD-NLS without overlay in order to allow comparison with Figure 1C. Scale bar represents $1 \mu \mathrm{m} \mathrm{in} \mathrm{A)} \mathrm{and} \mathrm{B),} 100 \mathrm{~nm}$ in C) and $25 \mathrm{~nm}$ in (C inset). 


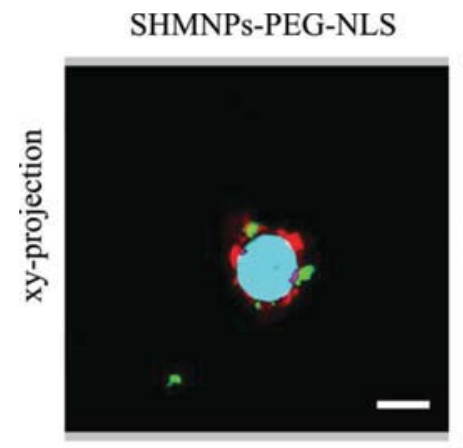

SHMNPs-PEG-cRGD
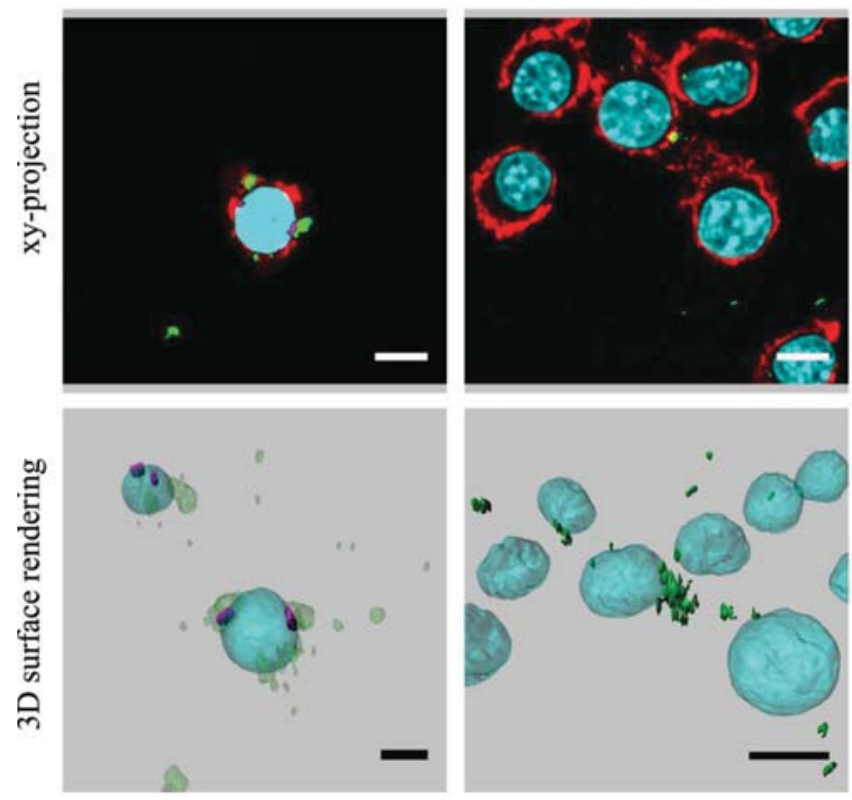

SHMNPs-PEG-cRGD-NLS
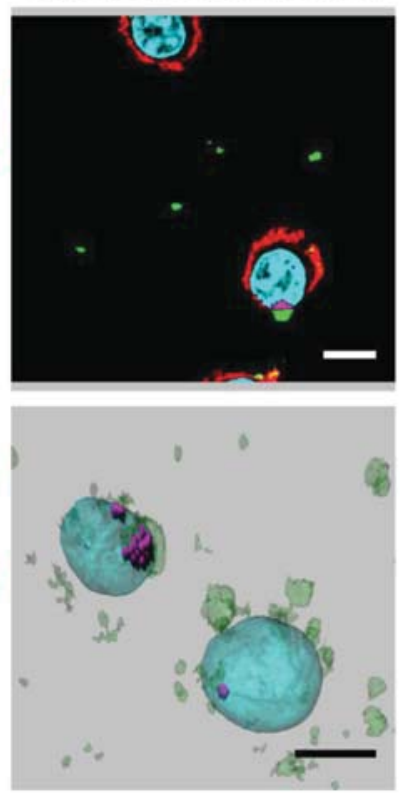

Figure 5. Laser scanning confocal microscopy (LSM) images showing the internalization of the NPs by J774A. 1 cells after 24 h exposure to SHMNPsPEG-NLS, SHMNPS-PEG-cRGD, or SHMNPs-PEG-cRGD-NLS at $20 \mu \mathrm{g} \mathrm{mL} \mathrm{m}^{-1}$. In the xy-projection, the red color corresponds to the cell membrane (F-actin) stained with phalloidin-rhodamine. In the 3D surface rendering of the xy-projections, the green fluorescence corresponds to the SHMNPsPEG-cRGD-NLS (Alexa488 fluorophore present on the surface of the NPs). The cyan color represents the cell nuclei (DAPI stain), while the green the different peptide sequences. The purple color evident in the 3D surface rendering images indicates an overlap between the cyan and green channels, indicative of NP association with the cell nuclei. Scale bars represent $10 \mu \mathrm{m}$.

localization was dependent of the specific peptide sequence. It must be noted here that it was not the goal of the study to enter the nucleus, but to uniquely characterize and identify them after cellular internalization. Although some studies have reported that NPs, which were much larger than the size of the nuclear pores, were able to penetrate the latter, ${ }^{[43]}$ nuclear internalization rates were almost negligible, ${ }^{[44]}$ while cargo NPs of larger diameters were found to predominantly accumulate around the pores of the nuclear envelope. ${ }^{[45]}$ When the cells were exposed to the SHMNPs-PEG-cRGD complex, NPs were also detected inside the cells (Figure $5 \mathrm{~B}$ ). It has been shown before that the cRGD ligand effectively binds to the integrins $(\alpha v \beta 3$ and $\alpha v \beta 5)$ on the J774A.1 macrophage cell surface. ${ }^{[4]}$ Figure 5B clearly shows NP uptake into the macrophages indicating that the cyclic peptide was efficiently coupled to the NPs' surfaces and that the cRGD ligand was effective in binding with the integrins known to be expressed on the surface of the J774A.1. ${ }^{[46]}$ On the occasion, the macrophage cells were exposed to SHMNPs-PEGcRGD-NLS, uptake of the NPs with a resultant association with the cell nuclei was observed (Figure 5C). Importantly however, whether or not the additional cRGD ligand induced a more prominent uptake compared to the SHMNPs-PEG-NLS could not be depicted from the LSM images. It is also worth noting that irrespective of the CRGD ligand being present on the surface of the NPs (Figure 5B), or not (Figure 5A), the NPs still gained access into the macrophage cells. This finding suggests a limited influence of the cRGD ligand in regards to the interaction of SHMNPs with immune cells. Finally, similar results for all three NP types were also observed with the (HeLa) epithelial cells, although, as highlighted previously, the interaction was less prominent with this barrier cell type compared to the phagocytic cells (Figure 6, Supporting Information).

\section{Conclusion}

The present study proves the reproducible functionalization of SHMNPs with specific peptides using a fast and cost-effective microreactor set-up. The uptake and intracellular fate of the synthesized NPs by two different subsets of cells (macrophages and epithelial cells) was confirmed by flow cytometry, LSM, TEM (conventional and elemental), and dark-field microscopy (with subsequent computational image restoration), which, when combined, could precisely characterize the NPs inside the cells and determine their intracellular localization. Furthermore, via this approach, it was possible to highlight the limited influence of the cRGD ligand on the interaction of SHMNPs with mammalian cells. Therefore, in conclusion, by incorporating a variety of state-of-the-art analytical and imaging approaches, it is possible to determine the specific effectiveness of surface peptide and ligand sequences upon multifunctional SHMNPs.

\section{Experimental Section}

Materials and Reagents: All chemicals were of analytical reagent grade and were used without further purification. Iron (III) nitrate nonahydrate $(98 \%)$, iron (III) chloride hexahydrate $(98 \%)$, iron (II) chloride tetrahydrate (99\%), APTES (99\%), TEOS (99\%), cysteine, sodium methoxide, and absolute ethanol (99\%) were supplied by 
Sigma-Aldrich (Switzerland). Ammonia (28\% in water), nitric acid $(65 \%)$, and disodium tetraborate decahydrate (98\%), methanol (99\%), sodium hydroxide (99\%), and sodium bicarbonate (98\%) were purchased from VWR International (Switzerland). Sodium citrate tribasic di-hydrate $(99.5 \%)$, glycine $(98 \%)$, and boric acid $(98 \%)$ were obtained from Fluka (Switzerland). FITC-Ahx (N-Terminal modification) labeled SV40 large T-antigen NLS peptide (PKKKRKVGC) was supplied by GenScript (USA). Cyclic RGD (cRGD: cyclo[Arg-Gly-Asp-D-Phe-Lys (Ac$\mathrm{SCH} 2 \mathrm{CO}$ )]) peptide was purchased from Peptides International (USA). Maleimide-PEG-carboxyheptyl-NHS (Mal-PEG-NHS) was supplied by NOF CORPORATION (Japan). AlexaFluor488 carboxylic acid, succinimidyl ester, mixed isomers, and SAMSA fluorescein (5-((2-(and3)-S-(acetylmercapto) succinoyl) amino) fluorescein) were purchased from Invitrogen (Switzerland). All reagents, fluorophores and antibodies used for cell culture experiments and microscopy were purchased from Sigma-Aldrich (Switzerland). D-9527 Sigma cellulose membrane dialysis tubing with a molecular weight cut-off of $12 \mathrm{kDa}$ was used for purification. All aqueous solutions were prepared with deionized water obtained from a Milli-Q system (resistivity $=18.2 \mathrm{M} \Omega \mathrm{cm}$, Millipore AG, Switzerland). The magnetic reactor was developed in co-operation with the Centre Suisse d'Electronique et de Microtechnique SA (CSEM). The $\mathrm{FeNdB}$ magnets were purchased from Maurer Magnets (Switzerland). $\mathrm{Ni} /$ Fe mesh was supplied by Alfa Aesar (Switzerland). The HPLC pump 64 was supplied by Knaeur AG and an ultrasonic tube transducer was obtained from Telesonic Ultrasonics (Switzerland).

Synthesis of Superparamagnetic Iron Oxide Nanoparticles: Alkaline coprecipitation of ferric and ferrous chlorides in aqueous solution was performed to obtain SPIONs, based on the technique described by Bee et al..$^{[47]}$ and van Ewijk et al. ${ }^{[48]}$ and previously reported by Chastellain et al. ${ }^{[23]}$ SPIONs were transferred to a $7.5 \times 10^{-3} \mathrm{M}$ sodium citrate solution following previously reported protocol by Khosroshahi and Ghazanfari, ${ }^{[49]}$ Abramson et al., ${ }^{[50]}$ and Digigow et al. ${ }^{[21]}$

Synthesis of Silica Hybrid Magnetic Nanoparticles: SHMNPs were synthesized based on the original technique reported by Stöber and Fink ${ }^{\left[{ }^{11]}\right.}$ and Barnakov et al. ${ }^{[52]}$ The specific procedure used to synthesize the SHMNPs was previously described by Digigow et al. ${ }^{[2]]}$ These SPION containing silica beads can be easily functionalized via their surface amine groups and are highly responsive to magnetic fields, which facilitates all purification and concentration steps during the surface functionalization process. ${ }^{[1]}$

Preparation of the Functionalized SHMNPs using a Magnetic Reactor: The magnetic microreactor was used as previously described by Digigow et al. $\left.{ }^{[3]}\right]$ The SHMNPs were loaded onto the magnetic reactor at a flow rate of $0.5 \mathrm{~mL} \mathrm{~min}{ }^{-1}$. Coating with polyethylene-glycol was accomplished by recirculating $5 \mathrm{~mL}$ maleimide-PEG-carboxyheptyl-NHS $\left(1.25 \mathrm{mg} \mathrm{mL}^{-1}\right.$ Mal-PEG-NHS in $20 \times 10^{-3} \mathrm{~m}$ borate buffer solution, $\left.\mathrm{pH} 7.5\right)$ over $0.5 \mathrm{mg}$ immobilized SHMNPs for $1 \mathrm{~h}$. The PEGylated NPs (SHMNPs-PEG) were then washed with $5 \mathrm{~mL}$ boric acid solution $\left(20 \times 10^{-3} \mathrm{M}, \mathrm{pH}\right.$ 6.8). Prior to peptide coupling to SHMNPs-PEG, $1 \mathrm{mg}$ CRGD was deprotected with sodium methoxyde $(10 \mu \mathrm{L}, 0.5 \mathrm{~m})$. A total of $10 \mu \mathrm{L}$ of the cRGD peptide solution was mixed with $0.5 \mathrm{~mL}$ AlexaFluor488 $\left(1 \mathrm{mg} \mathrm{mL}^{-1}\right)$ and allowed to react for $1 \mathrm{~h}$ at room temperature. The product CRGDAlexaFluor 488 was diluted in $5 \mathrm{~mL}$ borate buffer $\left(20 \times 10^{-3} \mathrm{M}, \mathrm{pH}\right.$ 6.8) to a final concentration of $0.2 \mathrm{mg} \mathrm{mL}^{-1}$. The dye-labeled NLS peptide was dissolved in $5 \mathrm{~mL}$ borate buffer to a final concentration of $0.3 \mathrm{mg} \mathrm{mL}^{-1}$. For peptide coupling, $5 \mathrm{~mL}$ NLS solution and $1 \mathrm{~mL}$ CRGDAlexaFluor 488 solution were circulated on immobilized SHMNPs-PEG in the magnetic reactor, as described before. Two additional particle types, that is, SHMNPs-PEG-NLS and SHMNPs-PEG-CRGD, respectively, which contained only one peptide were synthesized as controls. For the elution of the immobilized NPs, the magnets were released and buffer solution loaded again to elute the NPs suspension. In order to help elution, an ultrasonic tube transducer was placed directed over the reaction chamber wall and a high frequency of ultrasound was applied for $30 \mathrm{~s}$ three times. At the end, the sample was collected and stored in a fridge at $4{ }^{\circ} \mathrm{C} .{ }^{[12]}$ After particle purification, unreacted maleimide groups were quenched by adding $1 \mathrm{~mL}$ of $4 \mathrm{mg} \mathrm{mL}$ cysteine and allowed to react for $1 \mathrm{~h}$. The functionalized NPs were subjected to another purification step, eluted from the reactor, and redispersed in Milli-Q water. All NPs were characterized in terms of their size, zeta potential, and number of surface peptides.

Dynamic Light Scattering: DLS measurements were carried out at a fixed angle $\left(90^{\circ}\right)$ on a photon correlation spectrometer from Brookhaven equipped with a BI-9000AT digital autocorrelator. The NPs were dispersed in borate buffer $\left(20 \times 10^{-3} \mathrm{M}, \mathrm{pH} 7.5\right)$ and the concentration was set to 0.030 to $0.100 \mathrm{mg} \mathrm{mL}^{-1}$ for all measurements. The theoretical refractive index of magnetite of $2.42^{[53]}$ was used to calculate the numberweighted distribution from the raw-intensity weighted data.

Zeta Potential Measurements: Zeta potential measurements were performed on a Particle Size Analyzer 90 plus from Brookhaven Instruments, equipped with a BI-9000AT platinum electrode. Viscosity, refractive index, and dielectric constant were set for pure water at $25^{\circ} \mathrm{C}$ The electrode was cleaned for $5 \mathrm{~min}$ in an ultrasonic bath prior to each measurement and pre-equilibrated for $2 \mathrm{~min}$ in an aliquot of the sample before data acquisition. The samples were prepared as described above for the DLS measurements.

Nanoparticle Concentration: The concentration of the SHMNPs suspension was determined following the gravimetric method by Skoog et al. ${ }^{[54]}$ Briefly, $1 \mathrm{~mL}$ SHMNPs suspension was deposited on an aluminum plate and weighed using an analytical balance (XA205, Mettler Toledo). Then, the solvent was evaporated in an oven at $150^{\circ} \mathrm{C}$ for $24 \mathrm{~h}$. The dry SHMNPs were then weighed again, and the concentration of SHMNPs in the suspension was subsequently calculated.

Peptide Quantification: The average number of fluorescent peptides per NP was estimated measuring their fluorescence (excitation/emission wavelength of 496 and $520 \mathrm{~nm}$, respectively) emission in a 96-well plate (Victor3 plate reader, Perkin Elmer, USA). Briefly, a serial dilution of the fluorescent peptide was prepared and the standard curve was used to determine the concentration of peptides per functionalized NP using the mean diameter of the NPs obtained from DLS (data not shown).

Cell Culture: 1774A.1 mouse macrophage-like cells were cultured as previously described by Clift et al. ${ }^{[35]}$ The cervical carcinoma epithelia cell-line (HeLa) was cultured as previously described by Hirsch et al. ${ }^{[22]}$

Cytotoxicity: The ability for the SHMNPs-PEG-CRGD-NLS to cause a cytotoxic effect upon the 1774A.1 macrophage and HeLa cells was assessed by quantifying the level of lactate dehydrogenase (LDH) release, a cytosolic enzyme indicative of cell membrane damage. Both cell types were exposed to the SHMNPs-PEG-CRGD-NLS at 20 and $100 \mu \mathrm{g} \mathrm{mL}$ for $24 \mathrm{~h}$. The level of LDH in the cell supernatant was assessed via a diagnostic kit (Roche, Switzerland), as previously described in Griffete et al. ${ }^{[55]} \mathrm{LDH}$ data are presented as the mean \pm standard error of the mean (SEM) ( $n=3$ in triplicate). Statistical significance was assessed by using a Students $t$-test (R statistical programme; r-project.org). Data were only considered significant if $p<0.05$.

Laser Scanning Microscopy: To assess the uptake and localization of the SHMNPs-PEG-CRGD-NLS in both the macrophage and epithelial cell-lines, LSM was employed. In a 12-well plate, at a density of $2.5 \times 10^{5}$ cells $\mathrm{mL}^{-1}$ J774A.1 and HeLa cells were cultured upon glass coverslips (15 mm diameter) for $24 \mathrm{~h}$ at $37{ }^{\circ} \mathrm{C}, 5 \% \mathrm{CO}_{2}$. After the incubation period, each cell type was exposed to $0.5 \mathrm{~mL}$ of 20 or 100 $\mu \mathrm{g} \mathrm{mL} \mathrm{m}^{-1}$ SHMNPs-PEG-CRGD-NLS for 2,6 , or $24 \mathrm{~h}$ at $37{ }^{\circ} \mathrm{C}, 5 \% \mathrm{CO}_{2}$. After each exposure period, samples were washed once with phosphate buffered saline (PBS) and then fixed with 3\% paraformaldehyde (SigmaAldrich, Switzerland) in PBS for $15 \mathrm{~min}$ at room temperature. Following subsequent treatment with $0.1 \mathrm{~m}$ glycine solution in PBS, samples were washed once with PBS and permeabilized with $0.2 \%$ Triton X-100 in PBS for $15 \mathrm{~min}$ at room temperature prior to immunofluorescent staining.

Immnunofluorescent Stainning and Imaging by LSM: Samples were washed three times with PBS and treated with $250 \mu \mathrm{L}$ mix of a 1:50 dilution of phalloidin-rhodamine and 1:100 dilution of $4^{\prime}, 6$-diamidino2-phenylindole (DAPI stain) for $60 \mathrm{~min}$ at room temperature in the dark. These fluorescent labels were chosen in order to stain both the cell cytoskeleton and the cell nuclei concomitantly. After completion of the staining period, samples were washed a further three times with PBS. Coverslips were then inverted and mounted onto microscope slides using Glycergel (Dako, Carpinteria, USA) and incubated at $4{ }^{\circ} \mathrm{C}$, 
in the dark, for $24 \mathrm{~h}$ prior to being imaged by confocal LSM (Carl Zeiss 710, Germany). All images were captured using a $63 \mathrm{x}$ magnification (numerical aperture $=1.3$ ). In addition to this, the $z$-stack module was used to confirm the intracellular localization of the NPs. Following acquisition, all images were subsequently processed using the 3D multi-channel image software IMARIS (Bitplane AG, Switzerland). It is important to note that colocalization analysis was carried out with the "IMARISColoc" module. The selection of colocalized voxels is thresholdbased (full intensity range used) and yielded a new channel that was subsequently used for the graphical output.

Fluorescence-Activated Cell Sorting (FACS): To semiquantify the uptake of the SHMNPs-PEG-CRGD-NLS by each different cell type, one-color flow cytometry was used. J774A.1 and HeLa cells were seeded at $2.5 \times$ $10^{5}$ cells $\mathrm{mL}^{-1}$ in $5 \mathrm{~mL}$ Falcon tubes (BD Biosciences, USA) for $24 \mathrm{~h}$ in an environment of $37^{\circ} \mathrm{C}, 5 \% \mathrm{CO}_{2}$. Following the incubation period, each cell type was exposed to $0.5 \mathrm{~mL}$ of 20 or $100 \mu \mathrm{g} \mathrm{mL}-1$ SHMNPs-PEGCRGD-NLS for either 2,6 , or $24 \mathrm{~h}$ at $37^{\circ} \mathrm{C}, 5 \% \mathrm{CO}_{2}$. After each exposure period, samples were centrifuged at $1300 \mathrm{rpm}$ for $8 \mathrm{~min}$ in order to obtain a cell pellet. Following removal of the excess supernatant, the cell pellet was then washed by resuspension in $0.5 \mathrm{~mL}$ "FACS buffer" (consisting of PBS $+1 \%$ bovine serum albumin (BSA) (Invitrogen, $\mathrm{CH}$ ) $+0.1 \%$ sodium azide (Invitrogen, $\mathrm{CH}$ )). Samples were then immediately analyzed by flow cytometry (BD LSR Fortessa). All fluorescent signals were collected in logarithmic mode (4 decade logarithmic amplifier) and cell numbers per channel in linear mode. Both the macrophage and epithelial cell populations were identified by an electronic gate being placed around the forward and side scatter modes. A total of 10000 gated events were acquired for each sample. The fluorescent amplifiers of FL-1 and FL-2 were adjusted to ensure that the negative cell population appeared in the first logarithmic decade. An electronic marker was then placed at the limit of the negative control to denote all positive cell populations in the final three logarithmic decades. No compensation for spectral overlap was performed since it was not required for one-color flow cytometry (FITC channel (denoting the fluorescent characteristics of the SHMNPs-PEG-CRGD-NLS) was only used).

Fluorescence, Dark-Field Microscopy (CytoViva): To further confirm the presence of the SHMNPs-PEG-CRGD-NLS within J774A.1 macrophagelike cells only, a hyperspectral imaging dark-field microscope (CytoViva, Auburn, AL, USA) equipped with a 100x lens with adjustable iris aperture ( 0.7 for dark field, 1.3 for fluorescence) was used. The system was additionally equipped with fluorescence capabilities (DAPI, Alexa 488 and Texas Red). Briefly, J774A.1 cells were cultured at $2.5 \times$ $10^{5}$ cells $\mathrm{mL}^{-1}$ in a 12-well plate containing $15 \mathrm{~mm}$ diameter glass coverslips for $24 \mathrm{~h}$ at $37{ }^{\circ} \mathrm{C}, 5 \% \mathrm{CO}_{2}$. Following the culture period, cells were exposed to $20 \mu \mathrm{g} \mathrm{mL}-1$ SHMNPs-PEG-CRGD-NLS for $24 \mathrm{~h}$ in an environment of $37^{\circ} \mathrm{C}, 5 \% \mathrm{CO}_{2}$. Samples were then stained for the nucleus and cytoskeleton as well as prepared upon microscope slides for dark-field microscopy as previously described (please refer to the "immunofluorescent staining and imaging by LSM" section). When imaged by the CytoViva, the three fluorescence channels were recorded on a Exi camera (QImaging, Surrey, BC, Canada) using a mercury lamp light source and an exposure time of $500 \mathrm{~ms}$. The objective lens was set to a numerical aperture of 1.3. The hyperspectral data cube was achieved using a spectrophotometer (400 to $1000 \mathrm{~nm}$ ) and recorded on a Pixelfly camera (PCO AG, Kelheim, Germany) using a quartz halogen aluminum reflector light source at an exposure time of $250 \mathrm{~ms}$ per line (720 lines). For the dark-field data, the objective lens iris was closed to a numerical aperture of 0.7. No changes in focus or position were made between fluorescence and HSI recording. Since the Exi and Pixelfly cameras have different dimensions, the overlay between both datasets was performed manually using Image) software.

Transmission Electron Microscopy: To further confirm the intracellular localization of the SHMNP-PEG-CRGD-NLS in J774A.1 macrophagelike cells, TEM was employed. J774A.1 cells were cultured at $2.5 \times 10^{5}$ cells $\mathrm{mL}^{-1}$ in a 12 -well plate for $24 \mathrm{~h}$ at $37{ }^{\circ} \mathrm{C}, 5 \% \mathrm{CO}_{2}$ before being exposed to $0.5 \mathrm{~mL}$ of $20 \mu \mathrm{g} \mathrm{mL} \mathrm{mL}^{-1}$ SHMNP-PEG-CRGD-NLS for $24 \mathrm{~h}$ in an environment of $37^{\circ} \mathrm{C}, 5 \% \mathrm{CO}_{2}$. Samples were then prepared for conventional and elemental TEM as previously described by
Brandenberger et al.[56] All samples were then analyzed using a Tecnai F20 TEM (FEl, Eindhoven, The Netherlands) equipped with a GIF Tridiem energy filter and Ultrascan 1000 CCD camera (Gatan, Pleasanton, USA). Elemental TEM was performed as previously described by Brandenberger et al..56] Briefly, a three-window method (82 eV and $95 \mathrm{eV}$ pre-edge, $115 \mathrm{eV}$ post-edge) with a slit width of $10 \mathrm{eV}$ was used. The first pre-edge recording image (Figure 7A, Supporting Information) shows the darkfield energy loss at $82 \mathrm{eV}$ post-edge (second pre-edge not shown). The post-edge image is shown in Figure 7B (Supporting Information).

\section{Supporting Information}

Supporting Information is available

\section{Acknowledgements}

This work was financially supported by the Swiss National Foundation (PP00P2-123373/1), the Adolphe Merkle Foundation, and the University of Fribourg. The Dr. Alfred Bretscher fund and the Microscopy Imaging Center (Institute of Anatomy, University of Bern) are also acknowledged for the use of the Tecnai F20 TEM.

[1] D. Shi, Adv. Funct. Mater. 2009, 19, 3356

[2] C. Alexiou, R. J. Schmid, R. Jurgons, M. Kremer, G. Wanner, C. Bergemann, E. Huenges, T. Nawroth, W. Arnold, F. G. Parak, Eur. Biophys. J. 2006, 35, 446.

[3] H. Zhang, D. Yee, C. Wang, Nanomedicine 2008, 3, 83.

[4] P. Nativo, I. A. Prior, M. Brust, ACS Nano 2008, 8, 1644.

[5] N. Symens, R. Walczak, J. Demeester, I. Mattaj, S. C. De Smedt, K. Remaut, Mol. Pharm. 2011, 8, 1757.

[6] A. Figuerola, R. Di Corato, L. Manna, T. Pellegrino, Pharmacol. Res. 2010, 62, 126.

[7] B. F. Pan, D. X. Cui, Y. Sheng, C. G. Ozkan, F. Gao, R. He, Q. Li, P. Xu, T. Huang, Cancer Res. 2007, 67, 8156.

[8] R. He, X. G. You, J. Shao, F. Gao, B. F. Pan, D. X. Cui, Nanotechnology 2007, 18, 315601.

[9] Z. M. Li, P. Huang, X. J. Zhang, J. Lin, S. Yang, B. Liu, F. Gao, P. Xi, Q. S. Ren, D. X. Cui, Mol. Pharmaceutics 2010, 7, 94.

[10] J. Ruan, J. Ji, H. Song, Q. Qian, K. Wang, C. Wang, D. Cui, Nanoscale Res. Lett. 2012, 7, 309.

[11] J. Salaklang, B. Steitz, A. Finka, C. P. O'Neil, M. Moniatte, A. J. van der Vlies, T. D. Giorgio, H. Hofmann, J. A. Hubbell, A. Petri-Fink, Angew. Chem. Int. Ed. 2008, 47, 7857.

[12] B. Steitz, J. Salaklang, A. Finka, C. O'Neil, H. Hofmann, A. Petri-Fink, Bioconjugate Chem. 2007, 18, 1684.

[13] Wahajuddin, S. Arora, Int. J. Nanomed. 2012, 7, 3445.

[14] M. J. Pittet, F. K. Swirski, F. Reynolds, L. Josephson, R. Weissleder, Nat. Protoc. 2006, 1, 73.

[15] H. P. Khng, D. Cunliffe, S. Davies, N. A. Turner, E. N. Vulfson, Biotechnol. Bioeng. 1998, 60, 419.

[16] J. Wang, J. Wu, Z. Zhang, X. Zhang, Z. Pan, L. Wang, L. Xu, Ultrasound Med. Biol. 2006, 32, 147.

[17] M. Breunig, S. Bauer, A. Goepferich, Eur. J. Pharm. Biopharm. 2008, 68, 112.

[18] A. G. Tkachenko, H. Xie, Y. Liu, D. Coleman, J. Ryan, W. R. Glomm, M. K. Shipton, S. Franzen, D. L. Feldheim, Bioconjugate Chem. 2004, $15,482$. 
[19] Z. Chen, J. Deng, Y. Zhao, T. Tao, Int. J. Nanomed. 2012, 7, 3803.

[20] E. Ruoslahti, Annu. Rev. Cell Dev. Biol. 1996, 12, 697.

[21] R. G. Digigow, J.-F. Dechézelles, H. Dietsch, I. Geissbühler, D. Vanhecke, C. Geers, A. M. Hirt, B. Rothen-Rutishauser, A. Petri-Fink, J. Magn. Magn. Mater. 2014, 362, 72.

[22] V. Hirsch, C. Kinnear, M. Moniatte, B. Rothen-Rutishauser, M. J. D. Clift, A. Fink, Nanoscale 2013, 5, 3723.

[23] M. Chastellain, A. Petri, H. Hofmann, J. Colloid Interface Sci. 2004 $278,353$.

[24] J. Salaklang, A. Petri-Fink, Am. Inst. Phys., Conf. Proc. 2010, 1311, 8.

[25] C. M. Feldherr, R. E. Lanford, D. Akin, Proc. Natl. Acad. Sci. U.S.A. $1992,89,11002$.

[26] D. Weerasinghe, J. Cell Biol. 1998, 142, 595.

[27] A. C. M. Sinanan, J. R. A. Machell, G. T. Wynne-Hughes, N. P. Hunt, M. P. Lewis, Biol. Cell 2008, 100, 465.

[28] A. Schmidt, E. Caron, A. Hall, Mol. Cell. Biol. 2001, 21, 438.

[29] S. Finnemann, E. Rodriguez-Boulan, J. Exp. Med. 1999, 190, 861.

[30] K. Herve, L. Douziech-Eyrolles, E. Munnier, S. Cohen-Jonathan, M. Soucé, H. Marchais, P. Limette, F. Warmont, M.-L. Saboungi, P. Dubois, I. Chourpa, Nanotechnology 2008, 19, 465608.

[31] R. G. Digigow, J.-F. Dechézelles, J. Kaufmann, D. Vanhecke, H. Knapp, M. Lattuada, B. Rothen-Rutishauser, A. Petri-Fink, Lab Chip. 2014, 14, 2276.

[32] X. Xia, M. Yang, Y. Wang, Y. Zheng, Q. Li, J. Chen, Y. Xia, ACS Nano 2012, 6, 512.

[33] B. Kang, M. A. Mackey, M. A. El-Sayed, J. Am. Chem. Soc. 2010, 132, 1517.

[34] B. Alberts, Molecular Biology of the Cell, Garland Science, New York 2002.

[35] M. J. Clift, B. Rothen-Rutishauser, D. M. Brown, R. Duffin, K. Donaldson, L. Proudfoot, K. Guy, V. Stone, Toxicol. Appl. Pharmacol. 2008, 232, 418.

[36] X. Zhi, H. Fang, C. Bao, G. Shen, J. Zhang, K. Wang, S. Guo, T. Wan, D. Cui, Biomaterials 2013, 34, 5254.
[37] R. Weissleder, M. Nahrendorf, M. J. Pittet, Nat. Mater. 2014, 13 125.

[38] M. A. Dobrovolskaia, P. Aggarwal, J. B. Hall, S. E. McNeil, Mol. Pharm. 2008, 5, 487.

[39] N. Fairbairn, A. Christofidou, A. G. Kanaras, Phys. Chem. Chem. Phys. 2013, 15, 4163.

[40] D. Vanhecke, L. Rodriguez-Lorenzo, M. J. D. Clift, F. Blank, A. Fink-Petri, B. Rothen-Rutishauser, Nanomedicine 2014, (in press).

[41] M. J. Geisow, W. Evans, Exp. Cell Res. 1984, 150, 36.

[42] J. G. Huang, T. Leshuk, F. X. Gu, Nano Today 2011, 6, 478.

[43] N. Pante, Mol. Biol. Cell 2002, 13, 425.

[44] C. M. Feldherr, D. Akin, J. Cell Sci. 1997, 110, 3065.

[45] H. F. Lodish, Molecular Cell Biology, W. H. Freeman, New York 2000.

[46] A. S. Antonov, F. D. Kolodgie, D. H. Munn, R. G. Gerrity, Am. J. Pathol. 2004, 165, 247.

[47] A. Bee, R. Massart, S. Neveu, J. Magn. Magn. Mater. 1995, $149,6$.

[48] G. van Ewijk, G. Vroege, A. Philipse, J. Magn. Magn. Mater. 1999, 201, 31.

[49] M. Khosroshahi, L. Ghazanfari, Phys. E 2010, 42, 1824.

[50] S. Abramson, L. Srithammavanh, J.-M. Siaugue, O. Horner, X. Xu, V. Cabuil, J. Nanopart. Res. 2009, 11, 459.

[51] W. Stöber, A. Fink, J. Colloid Interface Sci. 1968, 26, 62.

[52] Y. A. Barnakov, M. H. Yu, Z. Rosenzweig, Langmuir 2005, 21, 7524.

[53] P. Patnaik, Handbook of Inorganic Chemicals, McGraw-Hill, New York 2003.

[54] D. A. Skoog, D. M. West, F. J. Holler, Fundamentals of Analytical Chemistry, Saunders College Pub., Fort Worth 1996.

[55] N. Griffete, M. J. Clift, A. Lamouri, R. G. Digigow, A. M. Mihut, A. Fink, B. Rothen-Rutishauser, H. Dietsch, Colloids Surf., A 2012, $415,98$.

[56] C. Brandenberger, M. J. D. Clift, D. Vanhecke, C. Mühlfeld, V. Stone, P. Gehr, B. Rothen-Rutishauser, Part. Fibre Toxicol. 2010, 7, 15. 